\title{
Review Article \\ The Effectiveness of the Feldenkrais Method: A Systematic Review of the Evidence
}

\author{
Susan Hillier ${ }^{1}$ and Anthea Worley ${ }^{2}$ \\ ${ }^{1}$ International Centre for Allied Health Evidence, Sansom Institute of Health Research, School of Health Science, \\ University of South Australia, P.O. Box 2471, Adelaide, SA 5001, Australia \\ ${ }^{2}$ School of Health Science, University of South Australia, P.O. Box 2471, Adelaide, SA 5001, Australia \\ Correspondence should be addressed to Susan Hillier; susan.hillier@unisa.edu.au
}

Received 16 December 2014; Revised 4 March 2015; Accepted 9 March 2015

Academic Editor: Cun-Zhi Liu

Copyright (C) 2015 S. Hillier and A. Worley. This is an open access article distributed under the Creative Commons Attribution License, which permits unrestricted use, distribution, and reproduction in any medium, provided the original work is properly cited.

\begin{abstract}
The Feldenkrais Method (FM) has broad application in populations interested in improving awareness, health, and ease of function. This review aimed to update the evidence for the benefits of FM, and for which populations. A best practice systematic review protocol was devised. Included studies were appraised using the Cochrane risk of bias approach and trial findings analysed individually and collectively where possible. Twenty RCTs were included (an additional 14 to an earlier systematic review). The population, outcome, and findings were highly heterogeneous. However, meta-analyses were able to be performed with 7 studies, finding in favour of the FM for improving balance in ageing populations (e.g., timed up and go test MD $-1.14 \mathrm{sec}, 95 \% \mathrm{CI}-1.78$, -0.49 ; and functional reach test MD $6.08 \mathrm{~cm}, 95 \%$ CI 3.41, 8.74). Single studies reported significant positive effects for reduced perceived effort and increased comfort, body image perception, and dexterity. Risk of bias was high, thus tempering some results. Considered as a body of evidence, effects seem to be generic, supporting the proposal that FM works on a learning paradigm rather than disease-based mechanisms. Further research is required; however, in the meantime, clinicians and professionals may promote the use of FM in populations interested in efficient physical performance and self-efficacy.
\end{abstract}

\section{Introduction}

The Feldenkrais Method (FM) was developed over a period of decades in the last century by Dr. Moshe Feldenkrais. He claimed the basis of the approach was founded in the human potential for learning how to learn [1]. As such, he operationalized an experiential process or set of processes, whereby an individual or a group could be guided through a series of movement- and sensation-based explorations. The purpose of these explorations was to practise the nonlinear process of sensing the difference between two or more options to achieve the stated movement task, and making a discernment about which may feel easier, that is to say, performed with less effort. These perceptual discernments are predicated on a judgement that is positive (pleasurable, easy, and with less effort) compared with experiencing a less favourable feedback signal such as pain, strain, or discomfort. Further to this, the participants are encouraged to generate many alternative movement solutions to the guided task to increase the opportunity for further distinctions and improvements to be made. Thus the process of intention, action, gaining feedback, making decisions, and reenacting with adaptations constitutes the learning framework in a somatic context [2].

The two modes of delivery that are offered to the public are either individual, manually directed lessons (functional integration, FI) or group, verbally directed classes (awareness through movement, ATM). The nomenclature for both reflects the fundamentals of the approach-that movement has to be based on a functional or meaningful intention for the system to engage and that by becoming aware of what and how we act (move) we become in a better place to choose an alternative behaviour (movement pattern) [3]. Both modes of delivery apply the same principles of perceptual exploration through movement that is passively and/or actively performed.

The method has been applied in varied domains across countries, from general education or children with learning issues to enhancing performance in sports and theatre. 
The clinical applications have received the most interest in the published literature because of the intuitive appeal of basing a health recovery process on a learning paradigm and because of the inherent fostering of self-efficacy that occurs particularly in a group setting.

In the climate of evidence-based practice in the health domain, any approach being offered to the public is being scrutinized for evidence of effectiveness and, if effective, for what type of benefit and of what magnitude for any clinical population. An earlier systematic review of the evidence for the method was published in 2005 by Ernst and Canter [4]. This review included six randomised controlled trials (RCTs) of low to moderate quality in populations such as people with multiple sclerosis, chronic low back pain, and neck issues. They concluded that there was promising evidence but its credibility was tempered due to the low number of studies, high level of clinical heterogeneity between studies, and methodological flaws. The methods employed by Ernst and Canter [4] were robust for the time; however, their risk of bias assessment used a now discarded tool (the Jadad) and their search covered until 2003. Therefore, it is timely to systematically update the evidence for the Feldenkrais Method with current review procedures.

This review had the aims of

(1) systematically identifying and appraising the evidence for the effectiveness of the Feldenkrais Method across domains;

(2) determining what is the nature and order of magnitude of any beneficial effects and for which population/s.

\section{Materials and Methods}

2.1. Criteria for Considering Studies for This Review. We employed systematic review methods based on the PRISMA guidelines [5].

2.2. Types of Studies. We considered all types of primary studies in the first instance in order to fully explore the potential populations and outcomes covered. In the final inclusion only studies with a random allocation and a stated control group were included. Any secondary researches (systematic and semisystematic reviews) found were not included, but rather their included studies were retrieved in full and added to the potential pool in order for all primary studies to be appraised with a consistent method.

2.3. Types of Participants and Outcomes. We included any population where there was an outcome of interest related to improvement in health and/or function.

2.4. Types of Interventions and Comparisons. Either form of Feldenkrais Method (functional integration or awareness through movement) was included as the sole approach for the intervention group. The comparison group could include placebo, inactive control, or an alternate method.
2.5. Search Methods for Identification of Studies. We searched the databases of AMED (Allied and Complementary Medicine), Embase Classic + Embase, Ovid MEDLINE(R), Cochrane, PsycINFO, PubMed, and Google Scholar from inception to July 2014. We considered all languages (the search was open to all listed journals irrespective of language) and publication status (we would include unpublished trials wherever found, e.g., through experts in the field or grey literature such as organizational websites).

The search terms included variations and combinations of methodology terms (such as randomised, trial, clinical, and controlled), with intervention terms such as Feldenkrais Method, (awareness through movement and functional integration). An example of the terms employed in the electronic search strategy is presented in Table 1.

From the generated lists from each database, duplicates were removed and the first high level sift was performed by one author based on title alone. The second level of review was performed by both authors and required retrieval of the abstract at the minimum. The retained studies were examined in full to confirm inclusion. Those excluded were recorded with reasons.

All retrieved studies were checked for additional references, and experts in the field were contacted to assist in identifying any further studies published or unpublished. Experts were provided from the membership of peak FM bodies (the Australian Feldenkrais Guild and the International Feldenkrais Federation) and were asked to supply further papers by email.

2.6. Data Collection and Analysis. Relevant data were extracted from each of the included studies using a standard trial summary sheet by one author and checked by the second. Data included author, date, study design, population sample, intervention, comparison, outcome measures, results, and comments. A risk of bias evaluation was also performed for each study by one author using standard Cochrane tables [26] with checking and data entry by the second author. Any disagreements were resolved by consensus, with a third party if necessary.

Where possible, data were extracted for meta-analyses. We planned to extract and analyse data to calculate individual and total effect sizes through odds ratios or mean differences (fixed effect or random effect if the studies were small and/or heterogeneous) and 95\% confidence intervals. Statistical heterogeneity would be evaluated based on visual inspection of forest plots and on the $I^{2}$ statistic. It was not anticipated that any other analyses would be possible (e.g., subgroup or publication bias) due to a paucity of studies.

If we found that meta-analyses were not possible, then results would be synthesized and reported narratively.

\section{Results}

3.1. Included Studies. The systematic search yielded over 1,300 initial titles for high pass screening. See Figure 1 for the PRISMA Flow diagram. With duplicates and obviously irrelevant titles removed, 124 records were considered at the 
TABLE 1: Example of search strategy.

\begin{tabular}{ll}
\hline Number & Searches \\
\hline 1 & (Clinical trial or randomised trial or controlled trial).mp. [mp = ab, hw, ti, sh, tn, ot, \\
& dm, mf, dv, kw, nm, kf, ps, rs, an, ui] \\
& (Feldenkrais or awareness through movement or functional integration).mp. [mp = \\
2 & ab, hw, ti, sh, tn, ot, dm, mf, dv, kw, nm, kf, ps, rs, an, ui] \\
3 & 1 and 2 \\
4 & Removing duplicates from 3 \\
\hline
\end{tabular}

TABLE 2: List of papers excluded with reasons.

\begin{tabular}{ll}
\hline Studies & Reason for exclusion \\
\hline Kirkby (1994) & Controlled trial \\
Bearman (1999) & Pre/posttest (no control) \\
Seegert (1999) & Controlled trial \\
Huntley (2000) & Systematic review \\
Dunn (2000) & Pre/posttest (no control) \\
Fialka-Moser (2000) & Commentary \\
Malmgren-Ohlsen (2001, & Controlled trial \\
2002, 2003) & Controlled trial \\
Kerr (2002) & Review \\
Emerich (2003) & Posttest (no control) \\
Junker (2003) & Review \\
Galantino (2003) & Review \\
Gard (2005) & Review \\
Mehling (2005) & Review \\
Liptak (2005) & Pre/posttest (no control) \\
Batson (2005) & Pre/posttest (no control) \\
Wennemer (2006) & Descriptive \\
Porcino (2009) & Review (assessment) \\
Mehling (2009) & Content analysis \\
Connors (2010) & Controlled trial \\
Connors (201la) & Pre/posttest (no control) \\
Connors (2011b) & Inquiry (phenomenological) \\
Mehling (2011) & Pre/posttest (no control) \\
Ohman (2011) & Review \\
Laird (2012) & Fntervention (not exclusively \\
Mehling (2013) & \\
Gross (2013) & Review \\
Webb 2013 & Prestest (no control) \\
\hline
\end{tabular}

abstract level by both authors, with an additional two studies provided from experts in the field (newly published, one RCT, one non-RCT). Seventy-seven abstracts were excluded at this stage because they were did not report an investigation of the FM and/or did not involve a trial of effect. Forty-seven fulltext articles were reviewed against the criteria and further 27 excluded with reasons noted in Table 2 .

Fourteen new RCTs were included along with the original six studies from the Ernst and Canter [4] review. See Table 3 for details of all included studies. From this total of 20 studies, there were seven studies sufficiently homogenous to allow for meta-analyses.

3.2. Description of Studies. Publication dates ranged from 1991 [12] to 2014 [25]. Populations under investigation in the included RCTs ranged from healthy volunteers [6, 12, 15$17,19,24]$, healthy ageing [21-23], institutional ageing [25], people with multiple sclerosis [7-11,13], eating disorders [14], myocardial infarct [18], and sleep bruxism [20]. Studies generally had small sample sizes with a mean of 40.8 participants (SD 23.5).

The nature of the Feldenkrais interventions also varied in delivery mode, intensity, and frequency. The predominant methods were single or multiple ATM lessons delivered either in a group or individually using audio recording. The comparison groups were most commonly an alternate form of therapy. Fourteen trials had active controls (such as relaxation classes or generic movement/balance classes) and six had a passive or inactive control (usual activities/no intervention).

Outcomes were also highly heterogeneous in keeping with the needs of the diverse populations and are listed in Table 3. The measures related to performance or activity outcomes (e.g., balance or dexterity), symptoms (e.g., pain, effort or mood) or were related to quality of life.

3.3. Excluded Studies. Table 2 summarises the list of studies (27) that were retrieved but excluded. Reasons for exclusion were predominantly around design: two were systematic reviews; five were controlled trials (not randomly allocated); eight had no control group; eight were nonsystematic reviews; one was not exclusively Feldenkrais in the intervention group; one was a content analysis of an intervention; one was a phenomenological analysis; and one was a commentary.

3.4. Risk of Bias in Included Studies. Risk of bias was high in most studies. Less than a quarter of the studies had adequate random allocation processes and only a third had blinding of outcome assessments. It has to be acknowledged that for trials requiring an intervention like Feldenkrais it may be difficult or inappropriate to expect blinding of therapists or even participants, though participants can be blinded to the intervention of interest if there is a plausible comparison group (such as a relaxation or other forms of movementbased class). Figures 2 and 3 summarize the risk of bias analysis. It can be seen that a definitive judgement could not be made in many cases as it could not be confirmed whether 


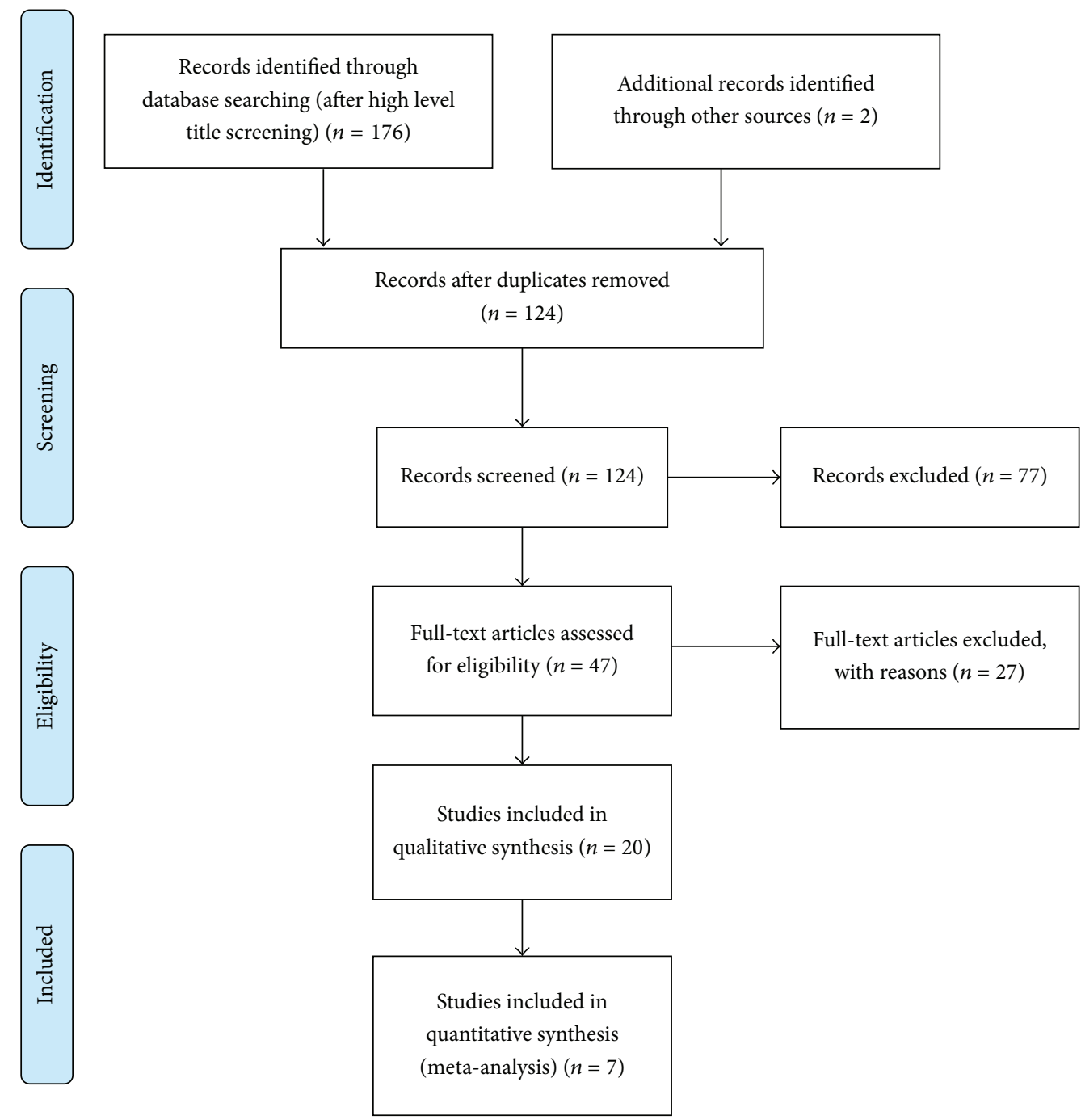

FIGURE 1: PRISMA flow diagram.

Random sequence generation (selection bias)

Allocation concealment (selection bias)

Blinding of participants and personnel (performance bias)

Blinding of outcome assessment (detection bias)

Incomplete outcome data (attrition bias)

Selective reporting (reporting bias)

Other biase
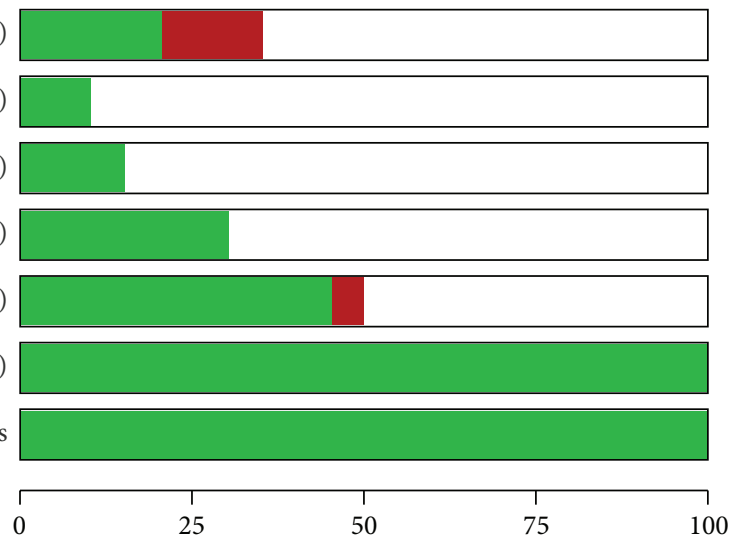

(\%)

Low risk of bias

Unclear risk of bias

FIGURE 2: Risk of bias graph: review authors' judgements about each risk of bias item presented as percentages across all included studies. 


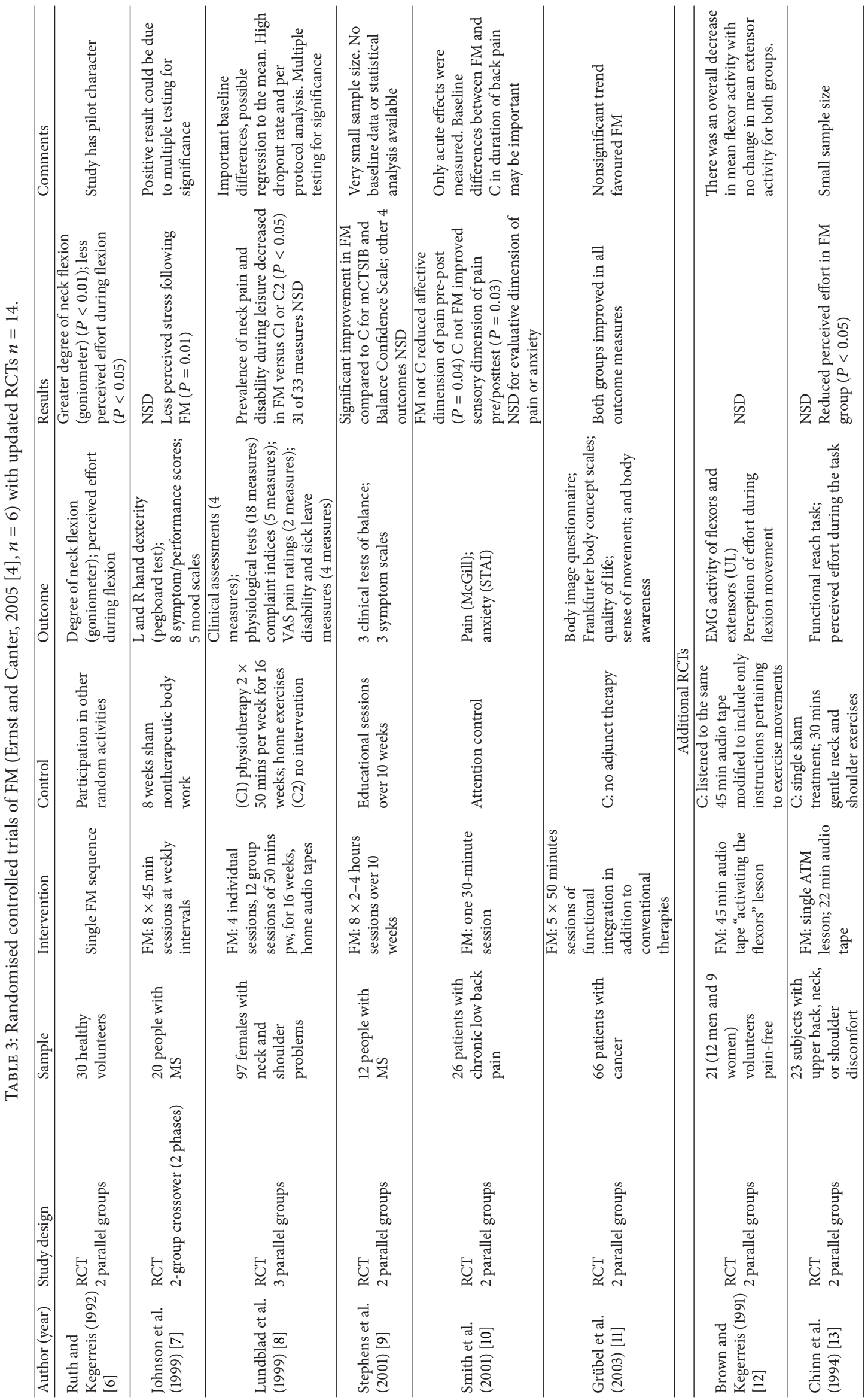




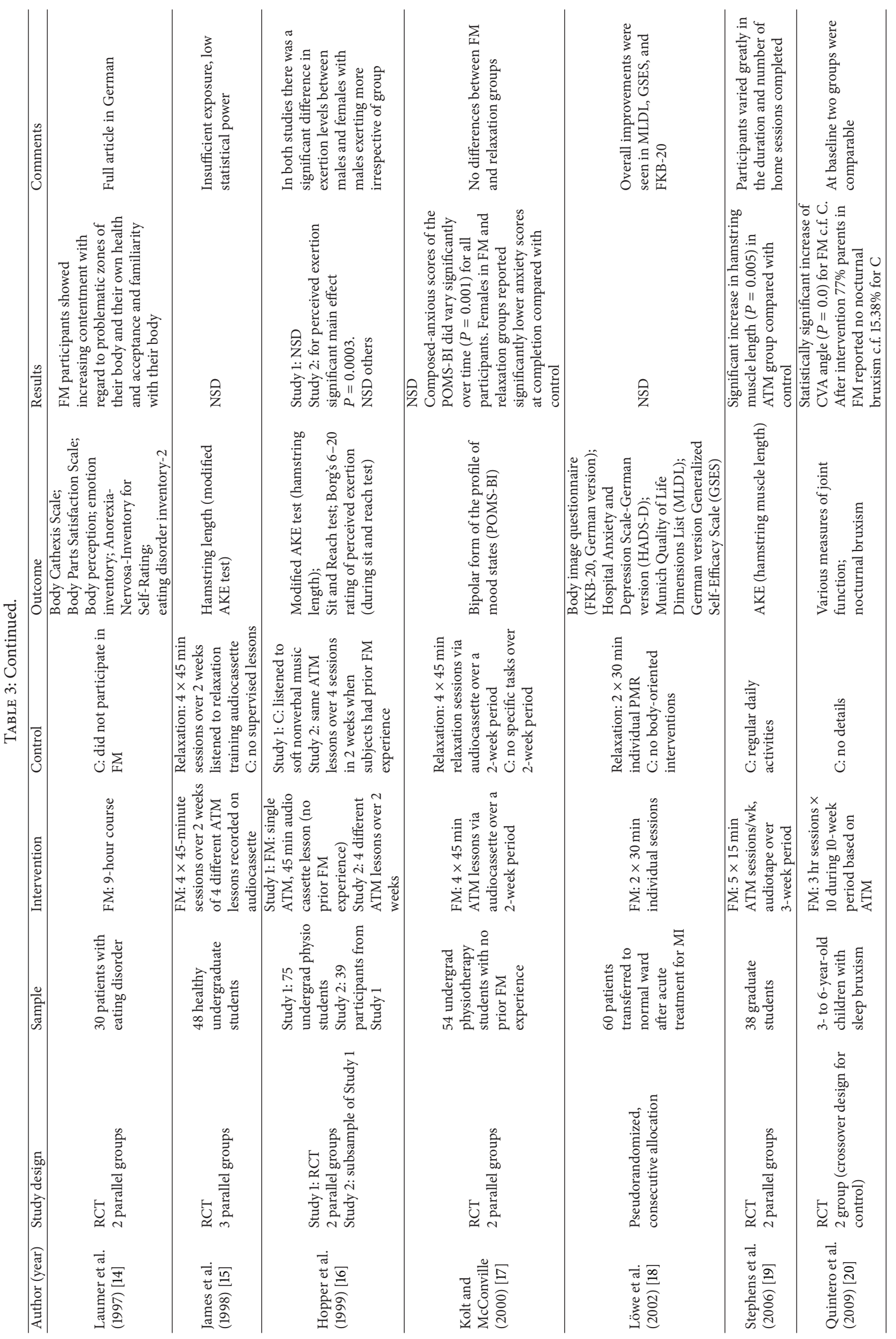




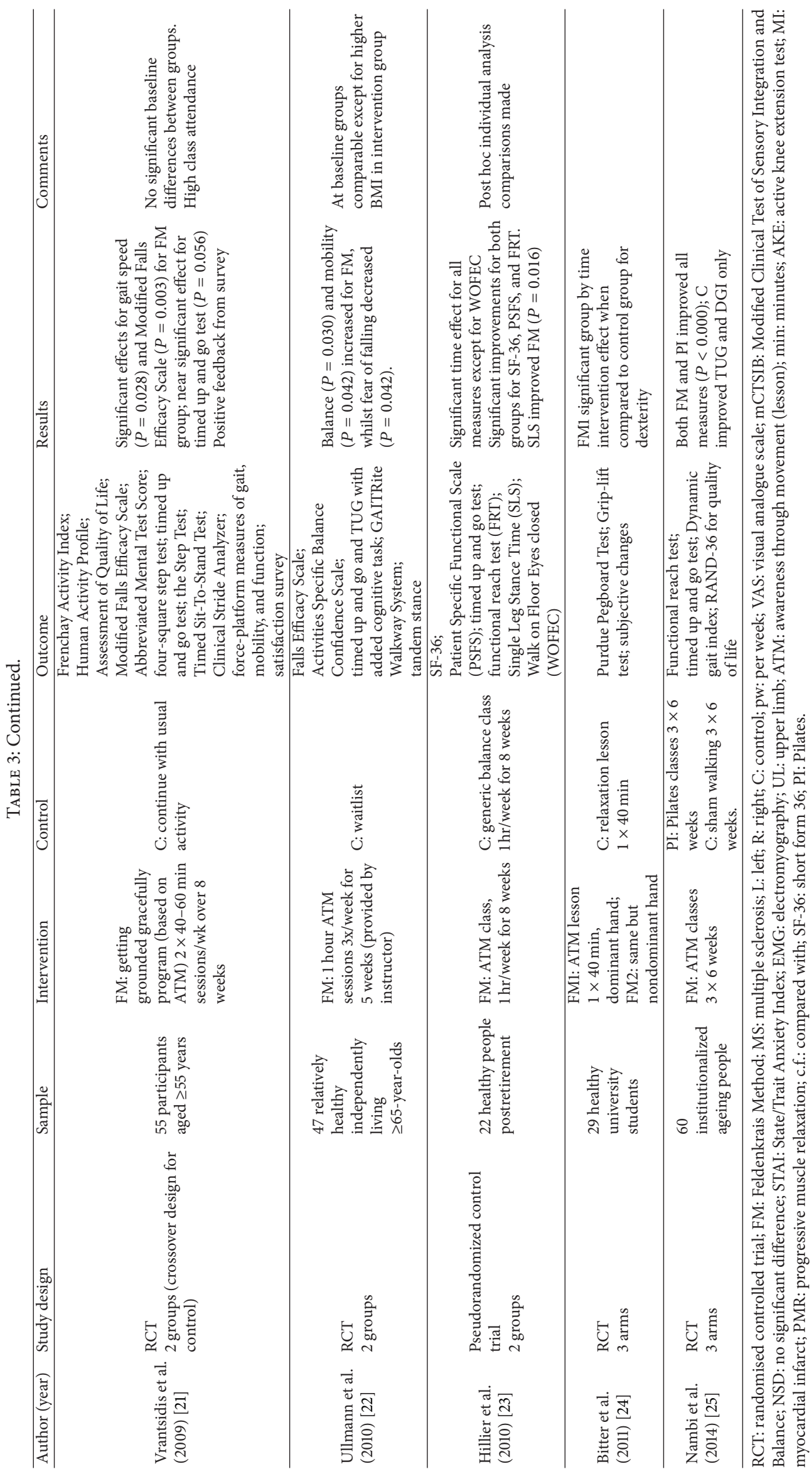




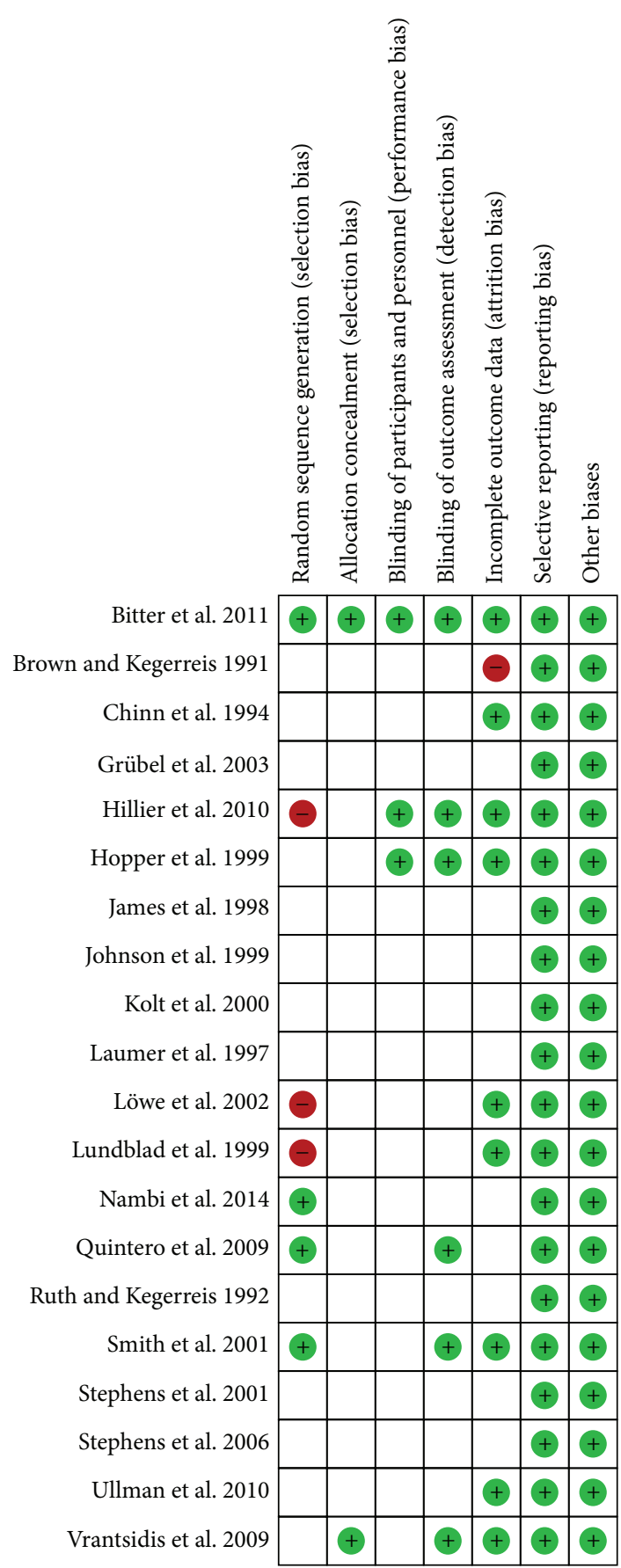

FIGURE 3: Risk of bias summary: review authors' judgements about each risk of bias item for each included study.

there was a clear risk of bias (given a red status) or whether the authors had simply not stated the process in sufficient detail for a judgement to be made; hence the risk of bias indicator was left blank.

3.5. Effects of Interventions. Sufficiently homogenous data (same population, intervention, comparator, and outcome measure) were able to be extracted to perform meta-analyses in the areas of balance training in ageing populations.
Four studies [21-23, 25] reported on the timed up and go assessment for balance and mobility, just failing to find in favour of Feldenkrais classes (Figure 4(a)); pooling postintervention measures gave a mean difference of $-0.78 \mathrm{~s}(95 \% \mathrm{CI}$ $-1.69,0.13), P=0.09$. However, heterogeneity was high $\left(I^{2}=\right.$ $49 \%)$. Therefore, a sensitivity analysis was performed as one study by Hillier et al. [23] compared Feldenkrais to another balance class whereas the other three studies compared the FM class to wait list control or no class. Removal of Hillier et al. [23] (Figure 4(b)) revealed a larger effect size with a mean difference of -1.13 (95\% CI $-1.7,-0.56), P=0.0001$, and heterogeneity reduced to a negligible level $\left(I^{2}=5 \%\right)$. It was also noted that Nambi et al. [25] had narrow outcome variability which led to a heavier weighting in the metaanalysis.

Two studies [21, 22] evaluated balance confidence using the Falls Efficacy Scale after FM classes (Figure 5). Pooled results trended in favour of the FM, however, failed to reach significance (MD 0.59, 95\% CI $-0.08,1.26 ; P=0.08$ ).

Two studies $[23,25]$ evaluated balance using the functional reach test after FM classes (Figure 6) - pooled results found in favour of the FM classes (compared to nothing or another generic balance class) with a mean difference of $6.08 \mathrm{~cm}$ (95\% CI 3.41,8.74), $P<0.00001$.

Meta-analysis was also able to be performed using three studies measuring the influence of FM classes on hamstring length in healthy populations $[15,16,19]$. The authors all reported the measure as an active knee extension test; however, on visual inspection, the results appeared heterogeneous in terms of magnitude and range; therefore, a standardized mean difference (rather than MD) was calculated. No significant effect was found after the intervention compared to control (SMD 0.15, 95\% CI -0.49, 0.79; $P=0.65$ ) and statistical heterogeneity was unacceptably high $\left(I^{2}=73 \%\right)$ (Figure 7).

Single randomised controlled studies reported statistically significant, positive benefits compared to control interventions and included the following:

(i) greater neck flexion and less perceived effort after a single FM lesson for neck comfort [6]; reduced prevalence of neck pain and disability in symptomatic women after FM (individual and group sessions compared to conventional care or home exercises) [8]; reduced perceived effort in FM group for people with upper torso/limb discomfort [13];

(ii) improved balance in people with MS after eight FM sessions [9];

(iii) improved body image parameters in people with eating disorders after a nine-hour FM course [14];

(iv) reduction in nocturnal bruxism in young children after 10-week course of FM lessons [20];

(v) improved dexterity in healthy young adults after a single session of FM class [24].

Seven of the 20 studies failed to show any superior positive effects of FM compared to other comparison modalities. See Table 3 for details. No studies reported adverse events. 


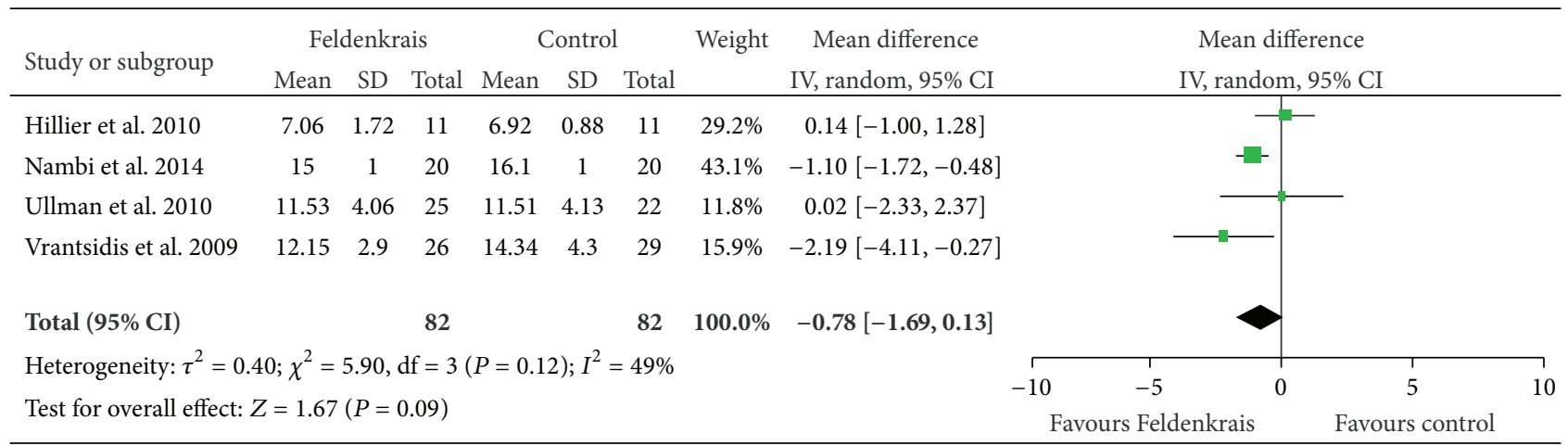

(a)

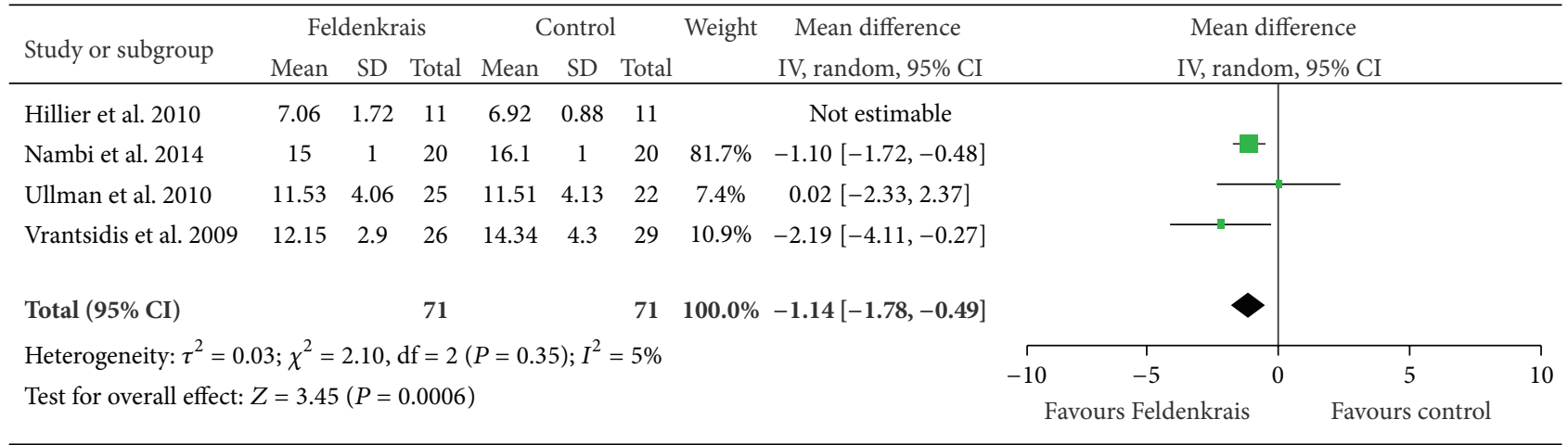

(b)

Figure 4: (a) Effect sizes of Feldenkrais versus control for the timed up and go test (measured in seconds; balance and mobility). (b) Effect sizes of Feldenkrais versus control for the timed up and go test (measured in seconds; balance and mobility) with Hillier 2010 removed (control group was alternate balance class).

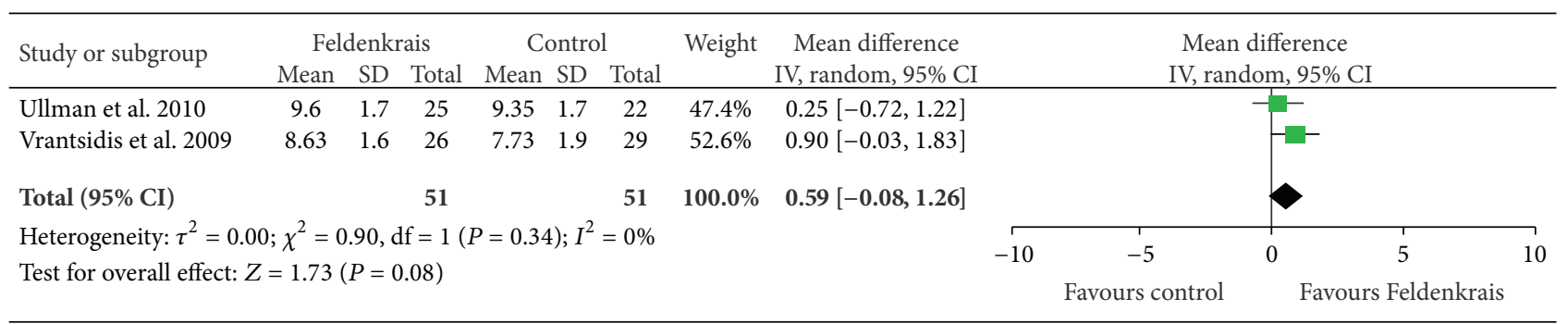

Figure 5: Effect sizes of Feldenkrais versus control for the Falls Efficacy Scale (balance confidence).

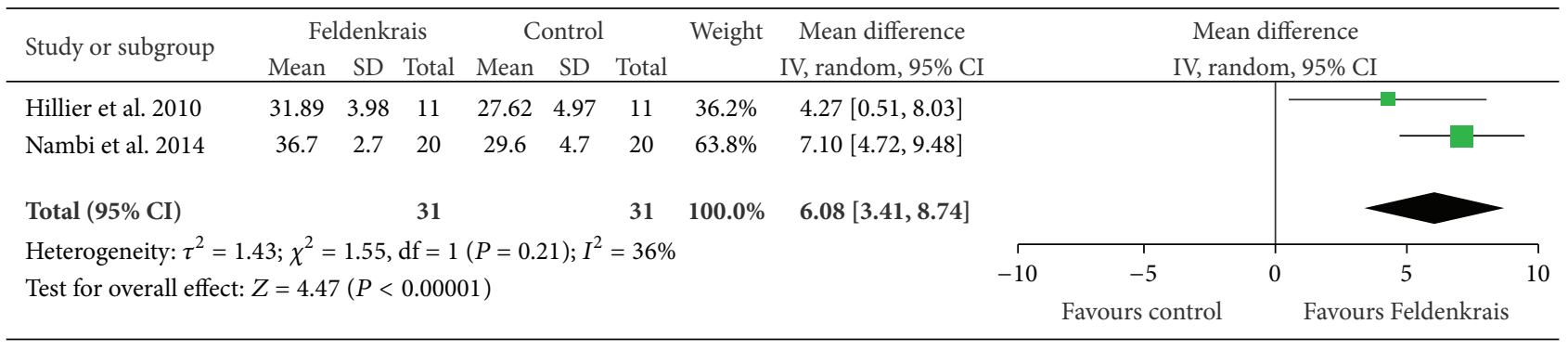

FiguRE 6: Effect sizes of Feldenkrais versus control for the functional reach test (measured in cm; balance). 


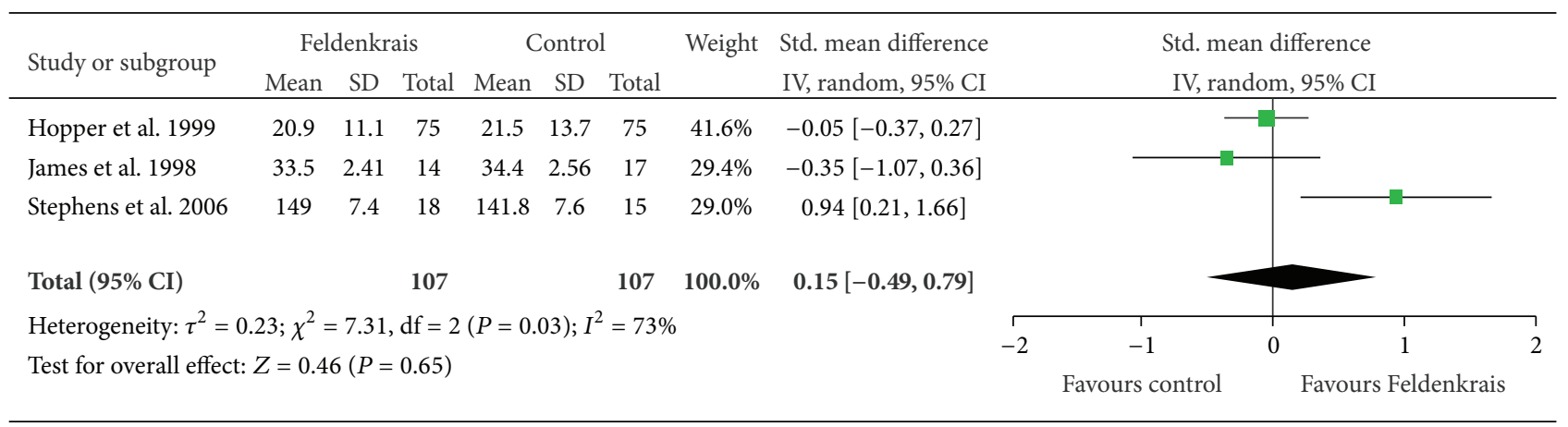

FIGURE 7: Effect sizes of the Feldenkrais Method on the active knee extension test.

\section{Discussion}

4.1. Summary of Main Results. The majority of the 20 included studies reported significant positive effects of FM in a variety of populations and outcomes of interest. A high risk of bias/poor methods reporting does temper the interpretation of these findings. The low amount of confirmed/reported adherence to best practice conduct of RCTs may be partially attributable to the age of the studies when knowledge in the area of trial conduct was less.

Nevertheless meta-analyses in the area of balance training in ageing populations were found in favour of the FM classes for clinical measures such as the timed up and go and functional reach tests. Both of these measures are predictive of falls risk. Whilst the TUG effect size was probably not clinically significant (1- to 2-second change), the functional reach test effect size would arguably indicate a clinically meaningful change (able to reach further $6 \mathrm{~cm}$ ).

Given the positive effects in particular outcome domains it is interesting to speculate on the mechanism of action of the FM; however, it is to be noted that this was not the purpose of the review. The favourable evidence for reduced perceptions of effort, improved dexterity, improved comfort and reduced bruxism all support the proposed mechanism of action via promotion of awareness, relaxation and more efficient action. Inconsistent results were found for improving hamstrings length indicating that a "relaxation" effect may be variable.

The populations varied in age and diagnosis indicating that a beneficial effect is possible across different domains; again this is consistent with the use of the FM in diverse populations and also consistent with the notion that it is not a healing or disease-specific mechanism of action but rather one based on more generic learning and self-improvement.

The findings of this updated review have strengthened since the 2005 review by Ernst and Canter [4]. We were also able to locate studies prior to 2005 that were not found by the original SR authors, presumably due to improved database access. As the previous authors reported, the studies are still highly varied and of often questionable quality. There is an ongoing issue of poor reporting, resulting in risks being judged "unclear"; it is unknown whether this hides undeclared risk or is simply an omission of reporting.

This review is not without its own limitations. This review includes all trials aimed at improving health and/or function so we have trials of healthy individuals as well as people with a clinical presentation. We have not included an analysis of publication bias, though we are confident that by using experts in the field and checking grey literature (organizational websites) we have made every effort to capture unpublished (negative) trials. We attempted to account for statistical heterogeneity and can conclude that the analysis for the timed up and go is more robust with the removal of Hillier et al. [23] (Figure 4(b)) because the comparator group differs from the other studies (alternate balance class versus no intervention) and secondly this study was pseudorandomized (allocation based on enrolment day). The question of inactive controls is vexed and permissible when proof of concept or pilot/phase 1 trials are being conducted. We encourage readers to take the stage of research and the design into account in their interpretation.

4.2. Implications for Practice. There is promising evidence that FM may be considered for balance classes in ageing populations, both as a preventative approach and for people at risk of falls. There is also some evidence for the use of FM where reduced effort, efficiency of movement, and awareness can play a part in reducing pain or discomfort.

4.3. Implications for Research. Further high quality research is required comparing FM to other modalities. Investigations should focus on the impact on self-efficacy, functional independence, and ease and efficiency of functioning, both as strategies for promotion of wellness and wellbeing and also for people with impairment who wish to improve their sense of ease. Mechanisms of effect also need to be investigated. Particular attention needs to be paid to the reporting of best practice trial design and to controlling for a potential placebo effect.

\section{Conclusions}

There is further promising evidence that the FM may be effective for a varied population interested in improving functions such as balance. Careful monitoring of individual impact is required given the varied evidence at a group level and the relatively poor quality of studies to date. 


\section{Disclosure}

Funding was from professional bodies involved in promoting FM but the bodies were not involved in the conduct of the review other than to identify experts within their membership to identify any missed/unpublished trials.

\section{Conflict of Interests}

The authors declare that there is no conflict of interests regarding the publication of this paper.

\section{Authors' Contribution}

Anthea Worley conducted the search and preliminary inclusions. Both authors contributed to the review of all papers and constructed the final report. One of the authors (Susan Hillier) was also author for two included studies; these were independently scrutinized.

\section{Acknowledgments}

The authors wish to acknowledge the financial assistance of the Australian Feldenkrais Guild and the International Feldenkrais Federation in supporting the costs of the search and appraisal.

\section{References}

[1] M. Feldenkrais, Awareness through Movement: Health Exercises for Personal Growth, Arkana, London, UK, 1990.

[2] K. A. Connors, M. P. Galea, C. M. Said, and L. J. Remedios, "Feldenkrais Method balance classes are based on principles of motor learning and postural control retraining: a qualitative research study," Physiotherapy, vol. 96, no. 4, pp. 324-336, 2010.

[3] P. A. Buchanan and B. D. Ulrich, "The Feldenkrais Method: a dynamic approach to changing motor behaviour," Research Quarterly for Exercise and Sport, vol. 72, no. 4, pp. 315-323, 2001.

[4] E. Ernst and P. H. Canter, "The feldenkrais method-a systematic review of randomised clinical trials," Physikalische Medizin Rehabilitationsmedizin Kurortmedizin, vol. 15, no. 3, pp. 151-156, 2005 (German).

[5] D. Moher, A. Liberati, J. Tetzlaff, D. G. Altman, and The PRISMA Group, "Preferred reporting items for systematic reviews and meta-analyses: the PRISMA statement," PLoS Medicine, vol. 6, no. 7, Article ID e1000097, 2009.

[6] S. Ruth and S. Kegerreis, "Facilitating cervical flexion using a Feldenkrais method: awareness through movement," Journal of Orthopaedic and Sports Physical Therapy, vol. 16, no. 1, pp. 2529, 1992.

[7] S. K. Johnson, J. Frederick, M. Kaufman, and B. Mountjoy, "A controlled investigation of bodywork in multiple sclerosis," Journal of Alternative and Complementary Medicine, vol. 5, no. 3, pp. 237-243, 1999.

[8] I. Lundblad, J. Elert, and B. Gerdle, "Randomized controlled trial of physiotherapy and Feldenkrais interventions in female workers with neck-shoulder complaints," Journal of Occupational Rehabilitation, vol. 9, no. 3, pp. 179-194, 1999.
[9] J. Stephens, D. DuShuttle, C. Hatcher, J. Shmunes, and C. Slaninka, "Use of awareness through movement improves balance and balance confidence in people with multiple sclerosis: a randomized controlled study," Neurology Report, vol. 25, no. 2, pp. 39-49, 2001.

[10] A. Smith, G. Kolt, and J. McConville, "The effect of the Feldenkrais method on pain and anxiety in people experiencing chronic low back pain," New Zealand Journal Physiotherapy, vol. 29, no. 1, pp. 6-14, 2001.

[11] R. Grübel, G. Erbacher, and A. Larisch, "Die wirksamkeit der feldenkrais-methode bei krebs-betroffenen," Erfahrungsheilkunde, vol. 52, no. 02, pp. 71-83, 2003 (German).

[12] E. Brown and S. Kegerreis, "Electromyographic activity of trunk musculature during a Feldenkrais awareness through movement lesson," Isokinetics and Exercise Science, vol. 1, no. 4, pp. 216-221, 1991.

[13] J. Chinn, D. Trujilo, S. Kegerreis, and T. Worrel, "Effect of a Feldenkrais intervention on symptomatic subjects performing a functional reach," Isokinetics and Exercise Science, vol. 4, no. 4, pp. 131-136, 1994.

[14] U. Laumer, M. Bauer, M. Fichter, and M. Helmut, "Therapeutische Effekte der Feldenkrais-Methode 'Bewusstheit durch Bewegung' bei Patienten mit Essstorungen [Therapeutic effects of the Feldenkrais Method (Awareness through Movement) in eating disorders]," Psychotherapie Psychosomatik Medizinische Psychologie, vol. 47, no. 5, pp. 170-180, 1997 (German).

[15] M. James, G. Kolt, J. McConville, and P. Bate, "The effects of a Feldenkrais program and relaxation procedures on hamstring length," Australian Journal of Physiotherapy, vol. 44, no. 1, pp. 49-54, 1998.

[16] C. Hopper, G. S. Kolt, and J. C. McConville, "The effects of Feldenkrais awareness through movement on hamstring length, flexibility, and perceived exertion," Journal of Bodywork and Movement Therapies, vol. 3, no. 4, pp. 238-247, 1999.

[17] G. S. Kolt and J. C. McConville, "The effects of a Feldenkrais (ATM) Awareness Through Movement program on state anxiety," Journal of Bodywork and Movement Therapies, vol. 4, no. 3, pp. 216-220, 2000.

[18] B. Löwe, K. Breining, S. Wilke, R. Wellmann, S. Zipfel, and W. Eich, "Quantitative and qualitative effects of Feldenkrais, progressive muscle relaxation, and standard medical treatment in patients after acute myocardial infarction," Psychotherapy Research, vol. 12, no. 2, pp. 179-191, 2002.

[19] J. Stephens, J. Davidson, J. DeRosa, M. Kriz, and N. Saltzman, "Lengthening the hamstring muscles without stretching using 'awareness through movement," Physical Therapy, vol. 86, no. 12, pp. 1641-1650, 2006.

[20] Y. Quintero, C. C. Restrepo, V. Tamayo et al., "Effect of awareness through movement on the head posture of bruxist children," Journal of Oral Rehabilitation, vol. 36, no. 1, pp. 18$25,2009$.

[21] F. Vrantsidis, K. D. Hill, K. Moore, R. Webb, S. Hunt, and L. Dowson, "Getting grounded gracefully: effectiveness and acceptability of feldenkrais in improving balance," Journal of Aging and Physical Activity, vol. 17, no. 1, pp. 57-76, 2009.

[22] G. Ullmann, H. G. Williams, J. Hussey, J. L. Durstine, and B. A. McClenaghan, "Effects of feldenkrais exercises on balance, mobility, balance confidence, and gait performance in community-dwelling adults age 65 and older," Journal of Alternative and Complementary Medicine, vol. 16, no. 1, pp. 97-105, 2010. 
[23] S. Hillier, L. Porter, K. Jackson, and J. Petkov, "The effects of Feldenkrais classes on the health and function of an ageing Australian sample: pilot study," The Open Rehabilitation Journal, vol. 3, no. 1, pp. 62-66, 2010.

[24] F. Bitter, S. Hillier, and L. Civetta, "Change in dexterity with sensory awareness training: a randomised controlled trial," Perceptual and Motor Skills, vol. 112, no. 3, pp. 783-798, 2011.

[25] G. Nambi, P. S. Trivedi, S. M. Momin, S. Patel, and D. P. Pancholi, "Comparative effect of Pilates and Feldenkrais intervention on functional balance and quality of life in ambulatory geriatric population: a randomized controlled study," International Journal of Health Sciences and Research, vol. 4, no. 3, pp. 71-77, 2014.

[26] J. P. T. Higgins and S. Green, Eds., Cochrane Handbook for Systematic Reviews of Interventions Version 5.1.0, The Cochrane Collaboration, 2011, http://www.cochrane-handbook.org/. 


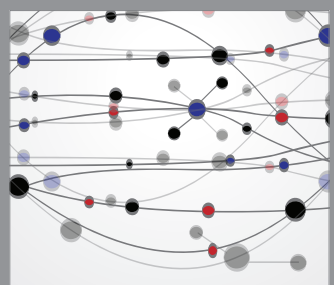

The Scientific World Journal
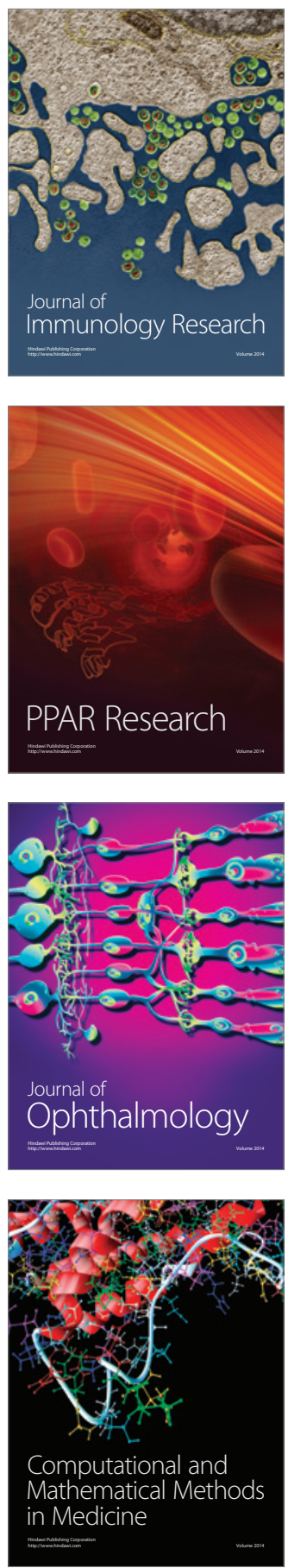

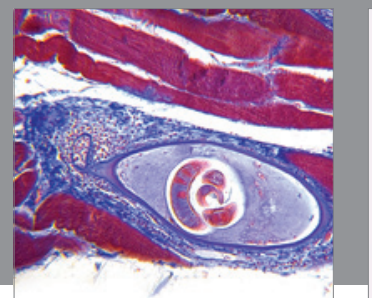

Gastroenterology

Research and Practice
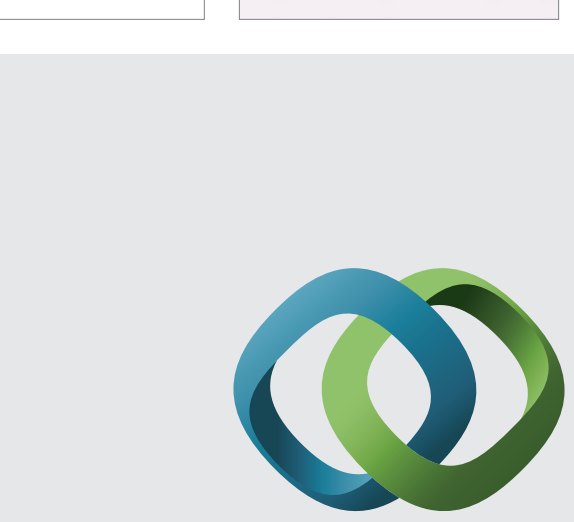

\section{Hindawi}

Submit your manuscripts at

http://www.hindawi.com
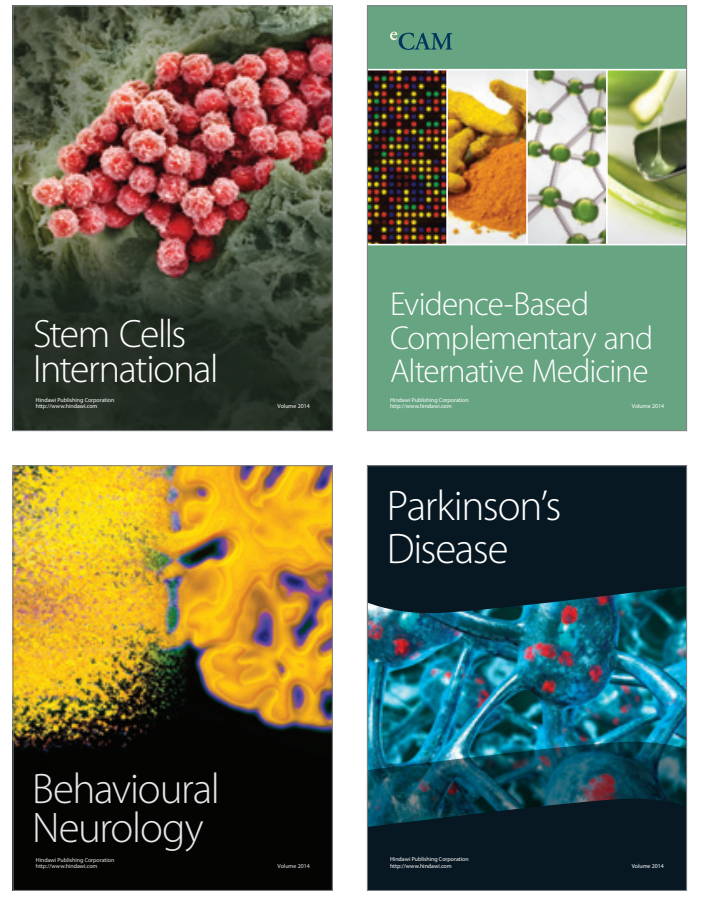
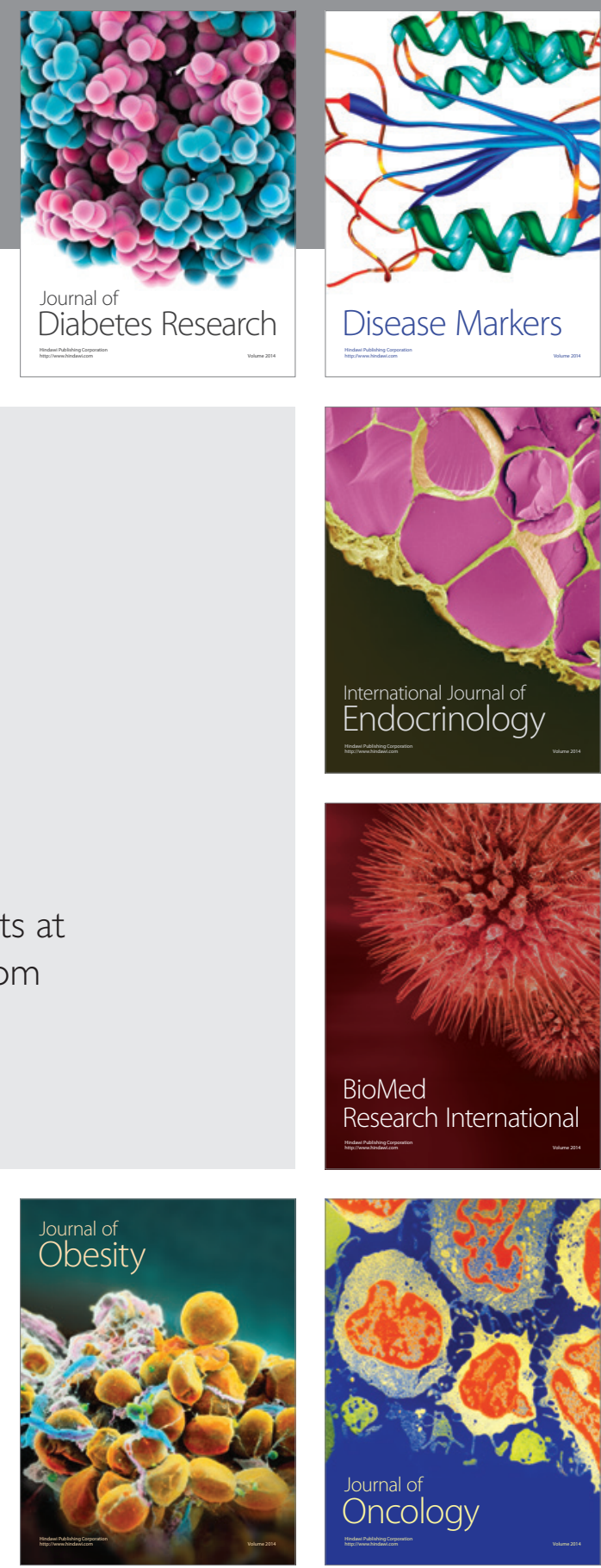

Disease Markers
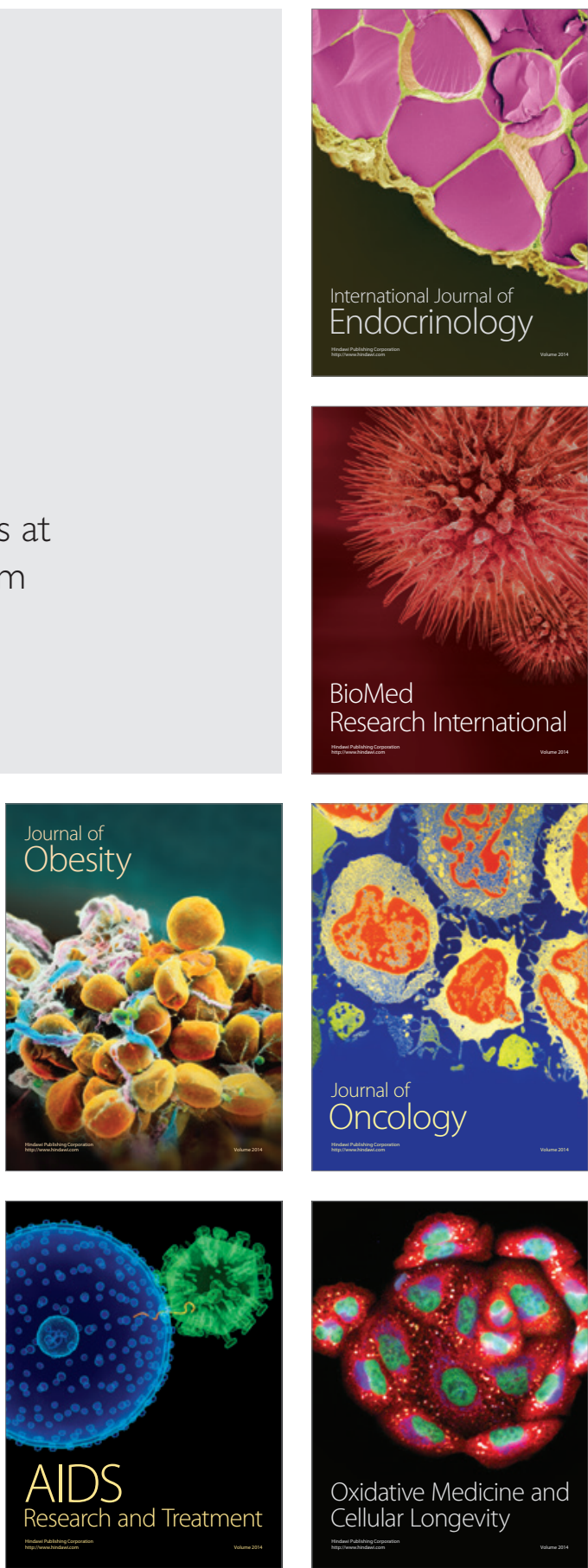